\title{
A Window into Occupant-driven Energy Outcomes: Leveraging Sub-metering Infrastructure to Examine Psychosocial Factors Driving Long-term Outcomes of Short-term Competition-based Energy Interventions
}

\begin{abstract}
Competition-based "energy saving" interventions are increasingly promoted as an effective strategy for reducing energy consumption in buildings with large occupant controlled electrical loads. However, the factors that drive energy savings in such interventions are not well understood, nor are the impacts of short-term competitions on long-term energy performance. A total of 39 8-occupant suites in a freshman residence hall were instrumented with "smart" electric meters, which recorded circuit-level electricity consumption at 15-minute intervals. During a three-week Fall 2014 competition, suites competed to reduce their overall electricity demand and achieved a $6.4 \%$ reduction in whole-building demand overall and a $12 \%$ reduction during hours of peak demand (from 12:00 to 19:00), despite peak seasonal temperatures and all-time record electricity demand. Analysis incorporating weather-normalized HVAC demand after the competition showed a significant "rebound" for a large portion of the suites (19), however 12 suites made further reductions, and the remainder maintained demand at the competition level. We compared energy data with self-reported survey data and identified self-efficacy beliefs, pro-environmental behaviors, and sense of affiliation with other residents of the hall as key factors distinguishing the suites with the greatest and most persistent reductions in demand from suites that maintained or increased demand.
\end{abstract}

\section{Keywords \\ Campus Building Energy Competitions, Energy Feedback, Demand Response, Psychosocial Factors, Building Electricity Sub-metering}




\section{INTRODUCTION}

In buildings where occupant-controlled electrical loads account for a large portion of whole-building demand, it is well known that improvements in building performance are dependent on systems which can effectively influence the participation and actions of occupants. Sociotechnical systems that visually present personalized energy feedback to occupants in real time are increasingly promoted as an effective technology for addressing this challenge by enabling competition-based "energy saving" interventions [10]. However, the factors that drive energy savings in such interventions are not well understood, nor are the impacts of such short-term interventions on long-term building energy performance. The objectives of the present study are to (1) examine long-term changes following a brief behavioral intervention to reduce energy use in a residential building; (2) investigate underlying psychosocial processes of change involved in the management of unregulated loads; and (3) explore the potential for demand response among unregulated loads.

\subsection{Significance}

Rising carbon dioxide $\left(\mathrm{CO}_{2}\right)$ emissions among the U.S. residential and commercial sector are projected through 2040, equating to 2150.4 million metric tons of $\mathrm{CO}_{2}$ [30]. To mitigate this predicted growth and its corresponding environmental and public health consequences, it is critical to manage energy demand. To achieve the $80 \%$ statewide greenhouse gas reduction target by 2050 mandated by California Executive Order S-3-05, the existing building stock must become $40 \%$ more energy efficient and all new construction must reach zero-net-energy by 2030 [8]. This objective can be facilitated using Demand Response (DR) capable buildings in which building management system (BMSs) control regulated loads. However, other building types, in which unregulated loads account for large portions of the total building load, present a significant challenge. Thus, although building infrastructure upgrades offer one approach for reducing building energy use, hardware upgrades alone cannot guarantee energy savings.

Occupant behavior, such as how frequently and intensively occupants choose to use air conditioners and other appliances, also impacts building energy consumption, and offers opportunities for savings. These opportunities are not trivial, with behavior change approaches resulting in savings comparable to technology-focused building retrofits [15]. Additionally, energy efficiency programs deployed in the U.S. from 2009-2012 cost 50$67 \%$ less per $\mathrm{kWh}$ than other power resources, including renewable energy, suggesting that such programs may serve as a financially viable resource [19]. Modifying occupant behavior, therefore represents a key opportunity for building energy management.

Recent work has leveraged behavioral science to better understand the theoretical underpinnings of energy use behavior [28], explore the effectiveness of interventions aimed at reducing energy and other resource use [1,2], propose models of sustainable energy technology acceptance [15] and better understand consumer adoption and optimal use of emerging smart grid technologies [25]. There is ample evidence to suggest that behavioral approaches can result in considerable energy savings, but less is known about how people change, or what the long-term impacts of these approaches are. 


\subsection{Residential energy conservation efforts}

A large body of research has investigated the impact of behavioral interventions on reducing energy consumption, particularly in residential buildings [2]. Briefly, based on the principles of operant learning theory, the approaches can be divided into two main categories: antecedents and consequences [27]. Antecedents are stimuli presented prior to the performance of a target behavior that serve as cues or facilitators of that behavior. Examples include information, prompts, modeling, and commitments / goal setting. Consequences are administered following a behavior, and the most common strategies used in residential energy reduction efforts include rewards and several types of feedback. A review of this literature is outside the scope of this paper, but these approaches have received considerable empirical support for their effectiveness in producing energy savings [2].

Creating new opportunities for modifying occupant energy use behaviors, "smart meters" collect high-resolution (e.g., 15-minute interval) usage data that can be shared with occupants in near real-time via various user-friendly platforms such as mobile devices and kiosks. This granular energy feedback can better enable building occupants to link their behaviors to their usage data and can motivate conservation. Advancing these efforts, findings suggest that building social tools into the delivery of energy feedback, for instance, socio-technical feedback systems that convey normative feedback [10], offers considerable promise in efforts to improve energy efficiency [1, 20, 22, 23].

\subsection{Demand response}

Reducing overall consumption is one important goal, and maintaining a reliable power supply is a second, equally important, objective. Even relatively brief lapses in power reliability have significant economic impacts. Estimates for annual economic losses from power interruptions include $€ 150$ billion among European Union businesses and $\$ 80$ billion in the United States [17]. Because energy demand varies by time of day, U.S. utilities are making efforts to curtail peak loads by managing demand, such as investing $\$ 700$ million annually in DR strategies (i.e., voluntary, time-delimited power usage curtailment events) to reduce peak load [30] as opposed to the traditional strategy of supplying additional generation, usually from higher-polluting energy sources [7]. Although DR forecasting models predict when, where, and how much energy will be used, solving the key problem of managing peak demand requires programs that encourage building occupants to make behavioral changes. There are two primary types of DR programs: (1) voluntary curtailment, which involves appealing to building occupants to temporarily curtail consumption by changing behavior in real time in response to alerts; or (2) direct control, in which occupants permit utilities to remotely control home equipment such as air conditioning units or thermostats. Voluntary curtailment programs typically use prompts and appeals to attempt to persuade occupants to curtail usage. Toward this end, utility-consumer connectivity must be enhanced. Programs must shift from a one-way, utility-to-consumer approach to a more interactive relationship, meeting consumers' needs to maximize program acceptance and enrollment levels.

\subsection{Energy reduction interventions in university buildings}

University campuses are important targets for energy management efforts for numerous reasons. First, they tend to be large energy consumers, with a considerable amount of 
energy consumed in residential buildings (e.g., [22]). Second, dormitories provide an excellent controlled setting for studying the efficacy of behavioral interventions because layouts are generally similar in size and basic electric infrastructure (e.g., [4]). Next, college students may be ideal candidates for behavioral interventions. Many students are making major life transitions, including relocating from childhood homes and forming new daily routines. Introducing new behavioral patterns during this time can increase the likelihood of establishing and maintaining new habits [34].

A handful of peer-reviewed studies have evaluated the effectiveness of electricity reduction interventions that applied combinations of the aforementioned intervention strategies to university housing occupants. Savings during interventions ranged from 8$19 \%$ based on different combinations of strategies [4, 5, 18, 21]. Only two studies examined long-term post-intervention impacts. Although they observed reduced levels of consumption up to 1-year follow-up, further research on this topic is warranted [18].

\subsection{Building energy competitions}

In recent years, many campuses have packaged combinations of intervention strategies as part of energy reduction competitions. Facilitated by socio-technical feedback systems, Campus Conservation Nationals $(\mathrm{CCN})$ has recently been established as an annual challenge in which colleges across North America compete to reduce energy and water consumption over several weeks [9]. Although these competitions appear to be effective in motivating energy reductions, with 109 campuses saving a combined total of 2.2 million kWh electricity in 2014, the majority of projects have not undergone peer review, leaving unanswered questions about their impacts. However, Senbel and colleagues [24] leveraged an ongoing CCN competition across six college campuses in British Columbia to study the combined impact of commitments, prompts, socio-technical feedback, civic engagement tasks, and individual- and group-level virtual incentives. During the 3 -week test period, electricity use in the test dormitory, which housed approximately 1800 students (approximately 11\% formally participated in the competition), dropped by $16 \%$, and remained at a reduced level over the remaining five months of the academic year (7\% below baseline).

A limited number of other competition-based energy reduction interventions have also been conducted in recent years outside the scope of CCN. For instance, Petersen and colleagues [22] investigated the impact of a competition framework that employed information, historical and normative feedback, and group-level incentives on energy and water conservation among undergraduate dormitory residents. Residents who received "high resolution" feedback (provided every 15 minutes) achieved savings of up to 55\% whereas "low-resolution" dormitories (feedback provided once/week) residents achieved a $31 \%$ reduction in usage, supporting feedback, particularly at high resolution, and incentives in producing short-term building energy savings. Additionally, at the University of Hawai'i, Brewer [6] implemented a 3-week energy competition among residents of several freshman dormitories that combined information, high-resolution feedback, and incentives. The winning team reduced energy use by $16 \%$ relative to baseline. Sintov et al. (in press) observed savings of $6 \%$ during a 3-week intervention that leveraged information, prompts, feedback, and incentives. The study was the first to include demand response events, which were met with mixed results. While most 
participants reported taking part, energy savings were observed during only one of two events in which occupants were asked to take part.

In 2015, Vine and Jones [33] reviewed results from 20 completed and ongoing energy reduction competitions, including both campus and non-campus competitions. The authors report that electricity savings were around 5\% during the program period for cases where savings were measured. However, the authors note that some programs received significantly higher savings on average (e.g. $21 \%$ ), or among the top percentage of buildings participating (e.g. 30\%), indicating the potential for deep savings from behavior-based strategies. In general, competitions that leveraged local, trusted messengers, continuously engaged participants, and leveraged social norms had some success in achieving reductions compared to baseline periods. While these results are promising and suggest the scalability of the competition approach beyond campus settings, persistence of savings beyond the competition timeframe, and the psychological processes, of change, remain largely unexplored.

\subsection{Theoretical motivators of behavior change}

Despite the success of previous behavior-based efforts in producing energy savings, identifying motivators of such change can be challenging. Although monetary incentives are traditionally viewed as a main driver of building occupant energy behavior, recent work shows that alternative approaches, such as those emphasizing health impacts, can motivate significantly more energy savings [4]. Several theories offer insights into motivators of change. For instance, the Theory of Planned Behavior postulates that behavior is proximally determined by intention to perform the behavior, which is more distally predicted by attitudes, normative beliefs, and perceived control (for details see [3]). In addition, under the framework of Self-Determination Theory, supporting an individual's autonomy, competence, and relatedness (connection with others) fosters motivation that can increase the likelihood of engaging in a variety of behaviors [11]. Notably, common to both models are social approval or sense of belonging, as well as a sense of agency or efficacy. More work is needed to determine whether energy reduction interventions may foster behavior change by tapping into these theoretically-based constructs.

\subsection{Gaps in the literature and study objectives}

What are the long-term impacts of behavioral interventions? Although the literature generally supports competition-based approaches in generating short-term energy savings among university residential building occupants, little is known about the long-term impacts of these efforts. Only two university-based studies have assessed postintervention changes in energy use, and both observed reduced consumption, up to one year post-intervention [18]. Longer-term studies that collect follow-up energy and selfreport data after interventions end are essential to investigate the durability of behavior changes and better understand the potential impacts of competitions and socio-feedback systems. The present study addresses this gap by examining follow-up data 2 months after the intervention ended. 
How can behavioral interventions be leveraged to improve building performance during peak hours? The literature on voluntary curtailment programs is relatively sparse. Despite the prevalence of these programs, many have not been evaluated or published, and among those that have, methodological limitations suggest areas for improvement (e.g. [29]). It is critical to investigate the impacts of these programs to better understand how they might fit into a broader portfolio of load management tools.

What are the psychological mechanisms underlying changes in energy use? Additionally, as highlighted by Sintov et al. (in press), building-level $\mathrm{kWh}$ consumption data, even at high resolution, does not allow us to see "behind closed doors" to individual-level behaviors or motivators of behavior. Such information would shed light on how and why occupant behavior changes. However, few studies have provided for assessment of energy data coupled with psychosocial data, for instance by using pre- and post- selfreport measures, so the psychosocial mechanisms underlying behavioral changes are not well understood. Only one study to date has provided preliminary evidence for how such changes map onto changes in energy use (Sintov et al., in press). To help refine future energy management efforts, it is critical to elucidate these processes - to understand how individuals change to achieve reductions. The present study builds on this emerging body of work. 


\section{METHOD}

The study was conducted at a large, private university in Southern California. All study procedures were approved by the University's Institutional Review Board. Participation was voluntary and participants provided informed consent.

\subsection{Participants}

The test building included 60 residential suites on 10 floors. Electricity and other utility bills were not paid directly by residents, but were integrated into a flat housing fee. With the exception of the first (ground) floor, each floor had six residential suites: one Resident Assistant (RA) suite, one single resident suite, and four group suites housing eight residents each.

\subsubsection{Inclusion and exclusion criteria.}

Individuals were eligible to participate in the study if they were residents of test building over the age of 18. Residents of RA and single suites were excluded because these suites were not equipped with digital electric meters. Faculty suite residents were also excluded. Of approximately 300 eligible residents, a total of 67 (22\%) residents from 16 suites on six floors completed a baseline survey, including the 48 who attended the kick-off meeting (mean $=1.7$ total surveys per suite, range $=0-8$ ). Because one of our key objectives was to examine changes over time, we included survey data from only residents who participated in both the baseline and follow-up surveys $(n=37)$. Drop-out analyses showed that those retained in the sample had significantly lower baseline group identification scores than those who were excluded; no other significant differences were found. In light of this finding and the high attrition rate, survey findings should be interpreted with caution.

\subsubsection{Samples used for analyses}

Excluding four individuals who answered validation questions incorrectly, the final sample size used for survey analyses was $n=33$. Using an intent-to-treat approach, we examined electricity data from all group suites except the faculty suite $(n=39)$. This provided for capturing any changes made by non-survey participants, who could have been exposed to intervention stimuli (e.g., kiosk was accessible to all residents and flyers were distributed to suites to be shared by all residents within a suite) and may have changed their behavior as well. Analyses looking at associations between changes in suite-level energy use and changes in self-report data are from a subset of 12 group suites that had residents with complete baseline and follow-up survey data.

\subsubsection{Survey participant characteristics}

Survey respondents were all freshmen with a mean age of 18.25. Approximately twothirds were females $(66 \%)$, and $76 \%$ identified as at least "somewhat" or more liberal political orientation. The ethnic breakdown of the sample was as follows: $11 \%$ African American, 13\% Asian American, 13\% Caucasian, 63\% Latino. 


\subsection{Materials and procedures}

Below is an overview of the study timeline:

Table 1. Study Timeline

\begin{tabular}{|l|l|l|}
\hline Week & Dates & Activity \\
\hline & 2014 & Instrumentation \\
\hline $1-2$ & August 24 - September 6 2015 & Baseline energy use monitored \\
\hline 3 & September 7- 10 2015 & $\begin{array}{l}\text { Kick off meeting; Baseline surveys } \\
\text { collected }\end{array}$ \\
\hline $4-6$ & September 11- October 1 2015 & Competition \\
\hline 7 & October 2-8 2105 & Follow-up surveys collected \\
\hline $8-16$ & & Post competition energy use monitored \\
\hline
\end{tabular}

Instrumentation (Figure 1). In the test building, digital electric meters were installed that collected energy consumption for each group suite in 15-minute increments. Data were transmitted via internet to the University's Facilities Management Services Energy Services Java Application Control Engine (JACE). Data were then transmitted to Lucid Design Group servers, processed, and transmitted by internet to the test building kiosk.

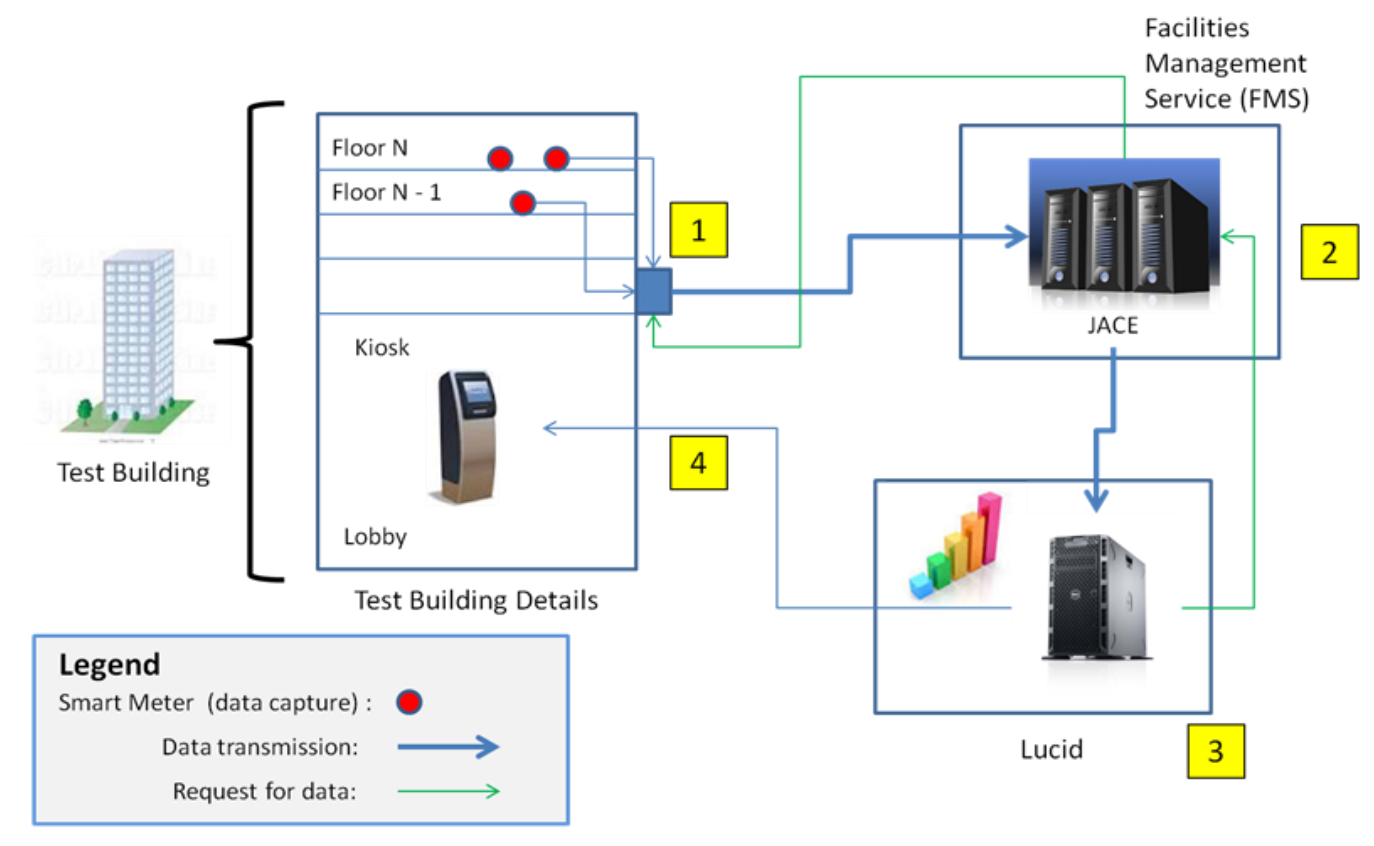

Figure 1. System diagram.

Baseline, Recruitment, and Kick-off. Suites' energy consumption was monitored for two weeks prior to the competition start without residents' knowledge to serve as the baseline. Flyers were then posted around the building to recruit residents to the kick-off meeting, during which they completed paper-pencil baseline surveys (also available online for several days to reach those who did not attend; see Survey Measures below for details). Study and Office of Residential Life (ORL) staff then explained the competition, responded to questions, and provided informational materials. 


\subsubsection{Intervention}

Flyers were delivered to each suite announcing the competition launch. Specific intervention strategies, which were selected based on prior empirical support and potential for replicability and scalability, included:

\subsubsection{Competition}

During the three-week competition, residents competed against one another to reduce their suite's and floor's energy consumption by performing any actions of their choosing.

\subsubsection{Incentives}

The suite and floor that reduced energy consumption by the largest percentage (compared to baseline measurements) were declared the winners. Members of the winning suite who completed baseline surveys were awarded movie passes ( $\$ 25$ value), and residents of winning floor (irrespective of baseline survey completion) won a floor party catered by a popular local eatery ( $\$ 300$ value).

\subsubsection{Feedback}

Historical and normative energy feedback were delivered via two methods: 1) A Kiosk and 2) Feedback reports. Located in the building lobby, the kiosk was an interactive touch-screen interface that provided high-resolution historical and normative energy feedback and updated suites' competition standings in near real time. Residents could interact with energy data by changing graph timescales and converting $\mathrm{kWh}$ to different units (e.g., $\mathrm{CO}_{2}$ emissions, hamburgers). To protect privacy, suite numbers were anonymized with a passcode known only to residents of each suite. Software was developed by Lucid Design Group and hardware was an Elo Touch Solutions 42" touchscreen monitor. After the first and second weeks of the competition, tailored feedback reports were delivered to each suite. The same information was also emailed to residents who provided email addresses on the baseline survey.

\subsubsection{Prompts}

Participants were also asked to take part in two 5-hour DR events, advertised as "Save Power Hours" and held on two separate days 10 days apart. The day before each DR event, flyers were distributed to prompt residents to participate in the next day's event. Flyers described a specific, two-step request, which included: (1) open windows the night before the event to pre-cool the building; and (2) minimize air conditioning use through the end of the event.

\subsubsection{Information}

To guide residents in their efforts, informational materials were distributed at the kick-off meeting. Extra copies were placed in the building lobby and were provided to RAs to distribute to residents not in attendance. A brochure and FAQ sheet summarized the competition, promoted the kiosk, listed important dates, and described rewards. A "Hot Tips" sheet provided suggestions for energy conservation behaviors residents could undertake. Behaviors were specifically selected based on major appliances and equipment installed in suites. 


\subsubsection{Follow-up survey}

Upon the end of the competition, flyers were posted (also emailed) to encourage participants to complete the follow-up survey, which was available online 24 hours per day for one week via Qualtrics, a secure survey software commonly used by university researchers.

\subsubsection{Survey measures}

Each survey required approximately ten minutes to complete. In addition to demographics, the baseline and follow-up surveys assessed frequency of energy conservation and other pro-environmental behaviors (PEBs) performed in the preceding weeks ("How frequently in the past few weeks have you...?"). We assessed energy and non-energy behaviors to provide for investigation of potential spill-over effects. The surveys also assessed select psychosocial variables from the Theory of Planned Behavior and Self-Determination Theory that we hypothesized would be most influenced by the intervention strategies applied, including: general self-efficacy beliefs ("I can usually handle whatever comes my way"); perceived general social norms (single item: "People in my general social environment support my efforts to save energy"); and perceived provincial social norms (single item: "People in my dormitory support my efforts to save energy"). Because we hypothesized that group identification would be associated with behavior change, particularly given the group incentives, we also assessed levels of group identification with target building residents (e.g., "I think of my residence hall as part of who I am"). The follow-up survey also assessed level of engagement with and exposure to competition stimuli. A validation question was included in each survey to detect random responding.

\subsection{Analyses}

\subsubsection{Building electricity demand}

The scope of the sub-metering infrastructure encompasses total electricity demand for all suites in the 10-floor "tower" section of the test building. Within this scope, all loads are occupant driven. Total suite-level demand is disaggregated at the circuit level into four load categories: "HVAC", "lighting", "plug", and "other". Heating, Ventilation and Air Conditioning (HVAC) for each suite is provided by multiple "through wall" Package Terminal Air Conditioner (PTAC) units, one for each of the four bedrooms and one for the shared living space. Suite-level outcomes were identified using an anonomizing code based on names of popular board games. All PTAC units are controlled by an accessible thermostat, enabling temperature set-points to be adjusted by occupants (the units are not controlled by occupancy sensors or timers). Windows in all rooms are manually operable. All kitchen appliance loads are recorded in the "other" load category.

\subsubsection{Initial suite-level differences}

Variations in baseline electricity demand among the 39 suites was also considered (figure 2). The average demand during the two-week baseline was $2.95 \mathrm{~kW}(\max =4.24$, $\min =$ 1.27 , st.dev $=0.74)$. Accounting for the eight occupants per suite leads to an average "per capita" demand of 370 Watts, (equivalent to nearly four 100-watt light bulbs on continuously per resident). Considering the relatively high degree of variation in electricity demand among suites prior to the competition, metrics of magnitude reduction 
and percent reduction were calculated to discern suites with low initial demand that achieved large percentage reductions from suites with high initial demand that achieved equivalent percentage reductions.

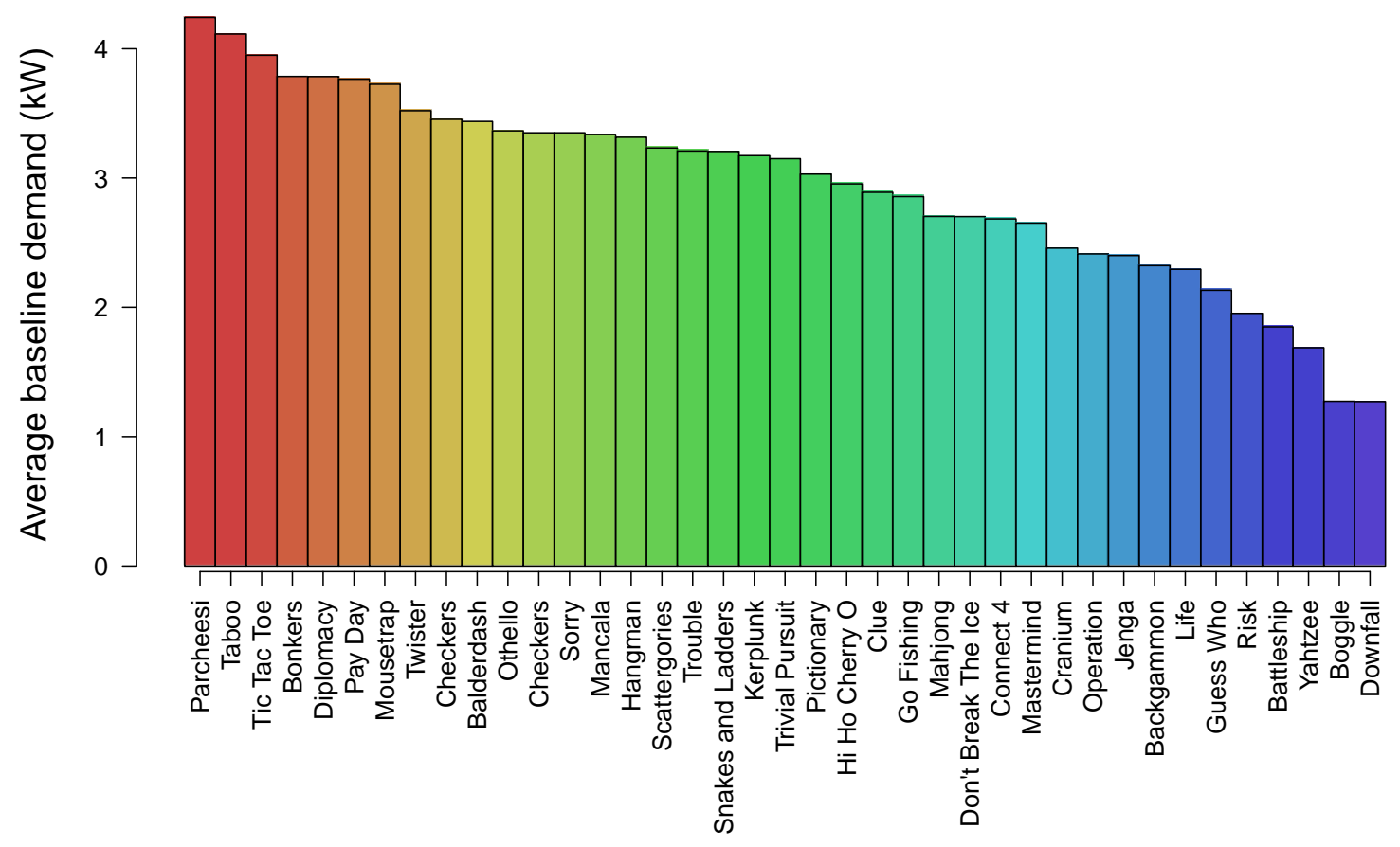

Figure 2. Average demand $(\mathrm{kW})$ during baseline period for each suite (game names are used as an anonomizing code for each suite).

\subsubsection{Load shape and peak demand}

The relative contribution of various electricity end uses to the building load shape, as well as time-of-use patterns were considered in developing the analysis procedures.

Figure 3 shows the disaggregated demand for each load category (Lighting, Plug, Other, and HVAC) during the two-week baseline period, using solid lines to separate each category. Among these categories, HVAC demand was found to be the dominant electricity end use, accounting for $65 \%$ of total demand on average during the baseline period. The average load shape during the 3 -week competition is shown with a dashed line, where the majority of the observed demand reduction was found to occur during the afternoon and evening hours (12:00 - 22:00). Because both overall demand reduction, and demand reduction during the critical afternoon hours of peak statewide demand are of interest, two timeframes were considered: all hours and a subset including only afternoon hours coinciding with periods of peak demand. Daily peak demand for the building occurs between the hours of 12:00 and 19:00 (indicated by vertical red lines), driven primarily by an increase in HVAC demand. 


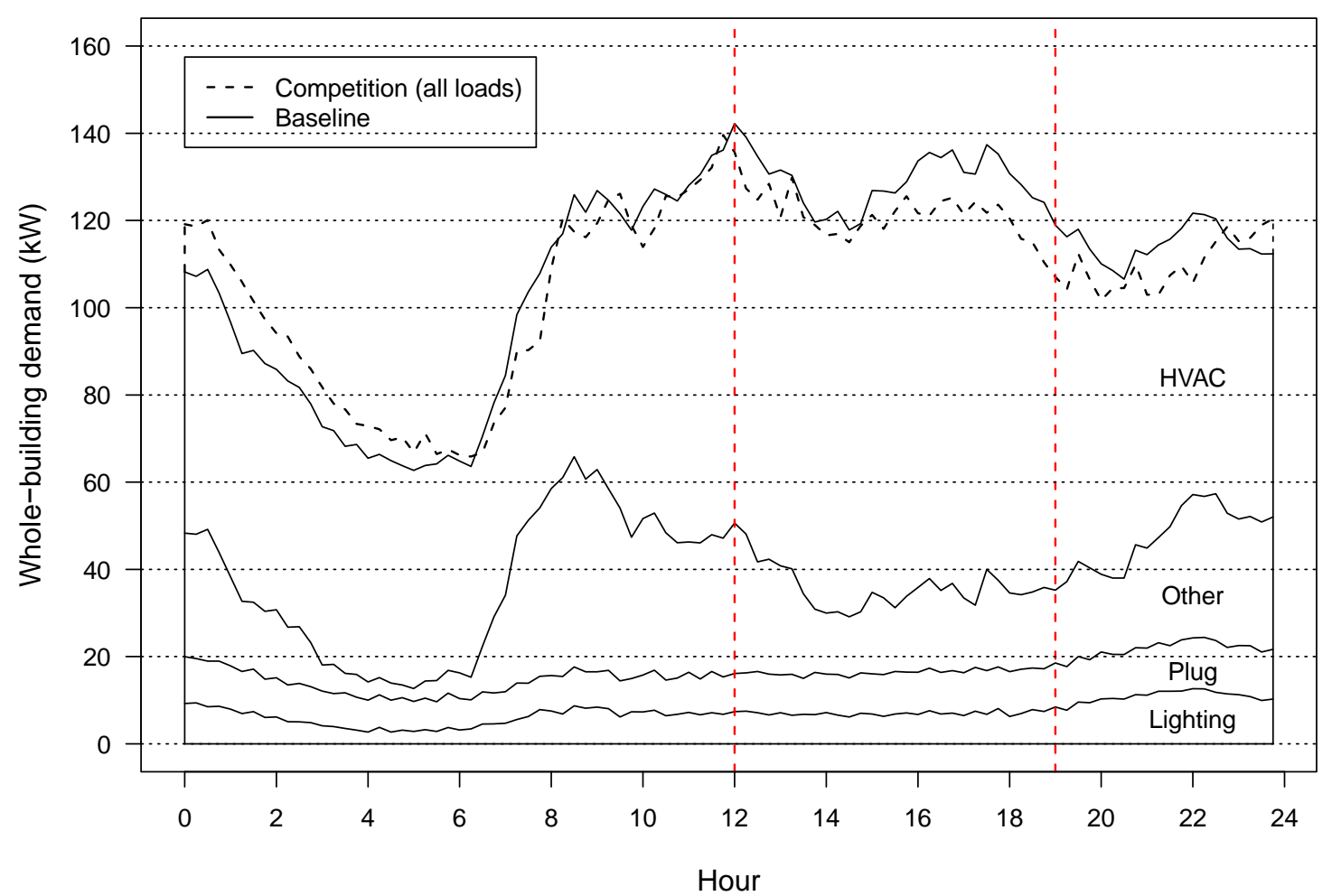

Figure 3. Test building load shape. Solid lines indicate the disaggregated demand for each load category (Lighting, Plug, Other, and HVAC) during the two-week baseline period. The average load shape during the 3-week competition is shown with a dashed line.

\subsubsection{Analysis procedure}

The baseline period was used as a benchmark to quantify changes in demand in the weeks during and after the competition. To examine the possibility of a short-term "snap back" in demand to baseline levels following the competition, our goal was to compare the two weeks following the end of the competition to the baseline period. To address the question of more long-term "rebound", we made similar comparisons using subsequent two-week intervals throughout the fall semester. One of the challenges with this approach is that suite-level demand, driven largely by air conditioning use during hot weather, was expected to vary based on outdoor temperatures, and this relationship can be readily seen in figure 4 and figure 5. Figure 4 presents summary statistics for the daily electricity demand of all 39 suites over the fall semester showing the two-week baseline period (day $236-250$ ), the three week competition period (solid vertical lines), and the 5 subsequent two-week periods which were examined to assess "snap back" and "rebound" effects after the competition ended. Figure 5 shows hourly outdoor temperatures during the same period. To evaluate the suitability of comparing competition and postcompetition outcomes to the baseline period, metrics of average daily Cooling Degree Days (CDD), and average outdoor temperatures were applied. Average daily CDD, and average daily temperatures were found to exceed the baseline period on average during the competition. Outcomes for the two weeks after the competition were found to be directly comparable to the baseline period. However, in the weeks following (days 289 to 
345), temperatures were generally cooler, and consequently the observed demand reduction is not directly comparable to the baseline. To address this issue, a weathernormalization technique was applied.

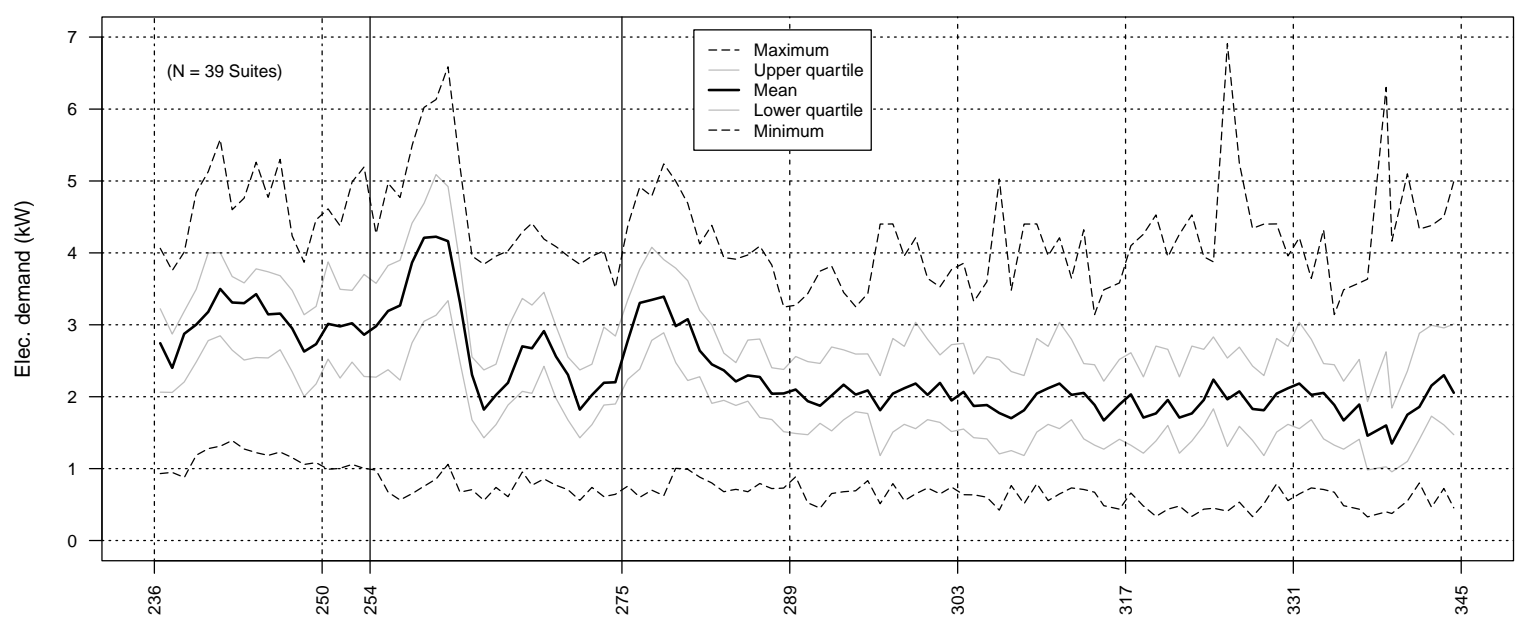

Figure 4. Suite-level trends in daily electricity demand.

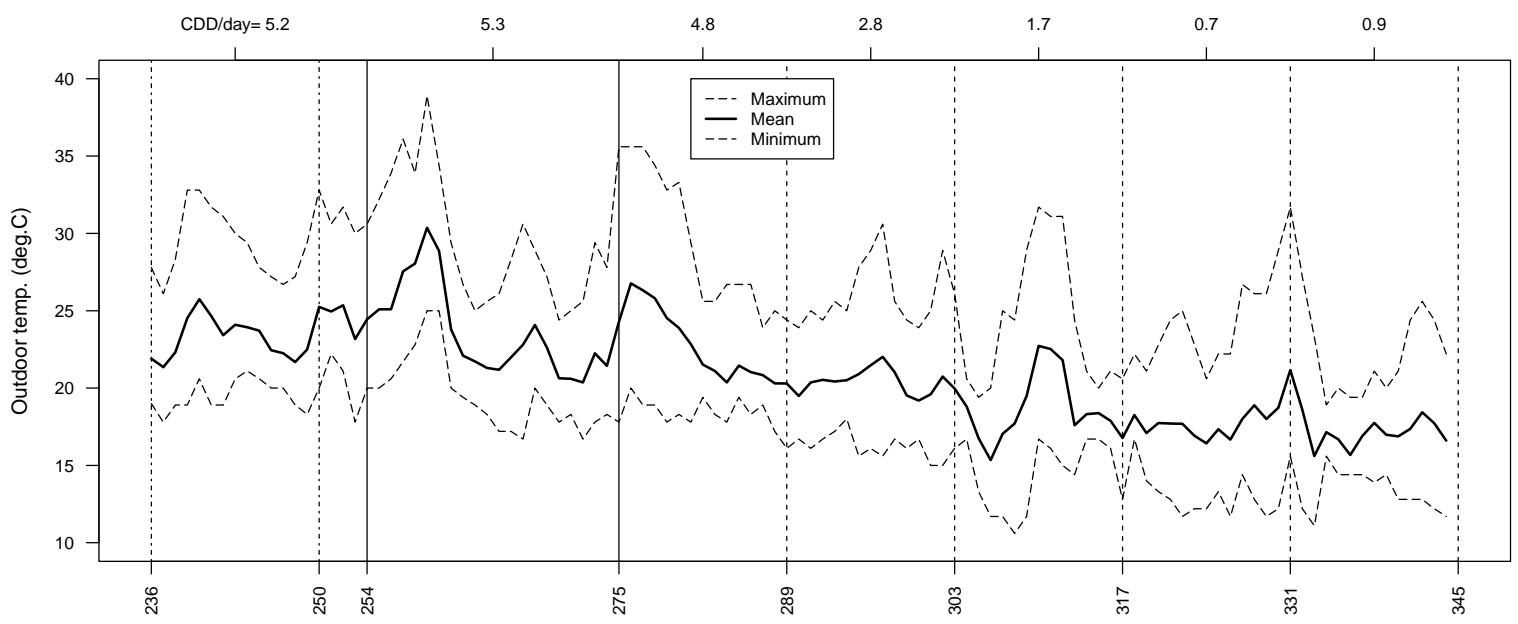

Figure 5. Summary of daily outdoor temperatures.

\subsubsection{Weather normalization}

Regression analysis revealed that average daily suite level HVAC electricity demand was linearly related to daily CDD, with demand increasing as daily CDD increased. A linear regression model was generated for each suite relating average HVAC electricity demand to CDD using baseline data. Models were then applied over the remainder of the fall semester to create a theoretical extension of baseline period HVAC utilization. Actual demand was then compared to modeled demand to discern the level of change, if any, that was not due to change in outdoor temperatures. 


\section{RESULTS}

Results are examined to evaluate the effectiveness of the competition framework that leveraged high-resolution historical and normative energy feedback, incentives, information, and prompts on short and long-term changes in energy use behaviors. An additional goal is to quantify and examine suite-level impacts and participation rates to assess the potential overall and peak-demand reductions achievable in future competitions if the outcomes of best-performance suites could be replicated by a greater number of suites. Participation rates, in this context, refer to the percent of total suites with a reduction relative to the baseline period. A final objective is to investigate change in both behavior and psychological correlates of behavior, and map these on to changes in short and long-term energy outcomes.

\subsection{Suite-level total electricity consumption}

In the following sections, results from analysis of suite-level interval data are used to examine the short and long-term changes in suite-level energy use. We hypothesized a significant reduction in energy consumption during the course of the intervention. Our analysis of post-competition changes was exploratory, and sought to test for "snap back" and "rebound effects" as defined in section 2.3. Outcomes during, immediately after, and in the four months after the competition are discussed in the following three sections.

\subsubsection{Competition vs. baseline}

Average electricity demand $(\mathrm{kW})$ during the weeks of the competition was $6.4 \%$ lower compared with the baseline period. This is notable given that the competition period had, on average, a greater number of CDD per day (9.70) relative to the baseline (9.13). Overall, 27 of the 39 suites (69\%) contributed to the observed demand reduction while the other 12 maintained or increased demand. Figure 6 presents the suite-level outcomes rank-ordered by the percentage change relative to the baseline period for all hours (colored). Shown in grey (adjacent) are the percent changes based on a comparison of peak demand (12:00 - 19:00) hours only. Negative values indicate reductions relative to the baseline period. Color assignments are the same as in figure 2, and are included to differentiate suites with high initial demand (e.g. $4 \mathrm{~kW}$ ) from suites with relatively lower initial demand (e.g. $1.3 \mathrm{~kW}$ ), where a relatively smaller percent reduction for the former may lead to a larger magnitude reduction compared with the latter.

Results based on a comparison of peak demand (12:00 - 19:00) hours only (grey) show a (13\%) overall demand reduction and an $82 \%$ participation rate (32 suites reduced demand relative to peak demand (12:00 - 19:00) hours during the baseline period), a significant improvement in both load reduction and participation. The level of demand reduction achieved during the peak demand interval is particularly notable for the competition period, given that it coincided with peak seasonal temperatures (figure 5) and all-time record peak electricity demand. 


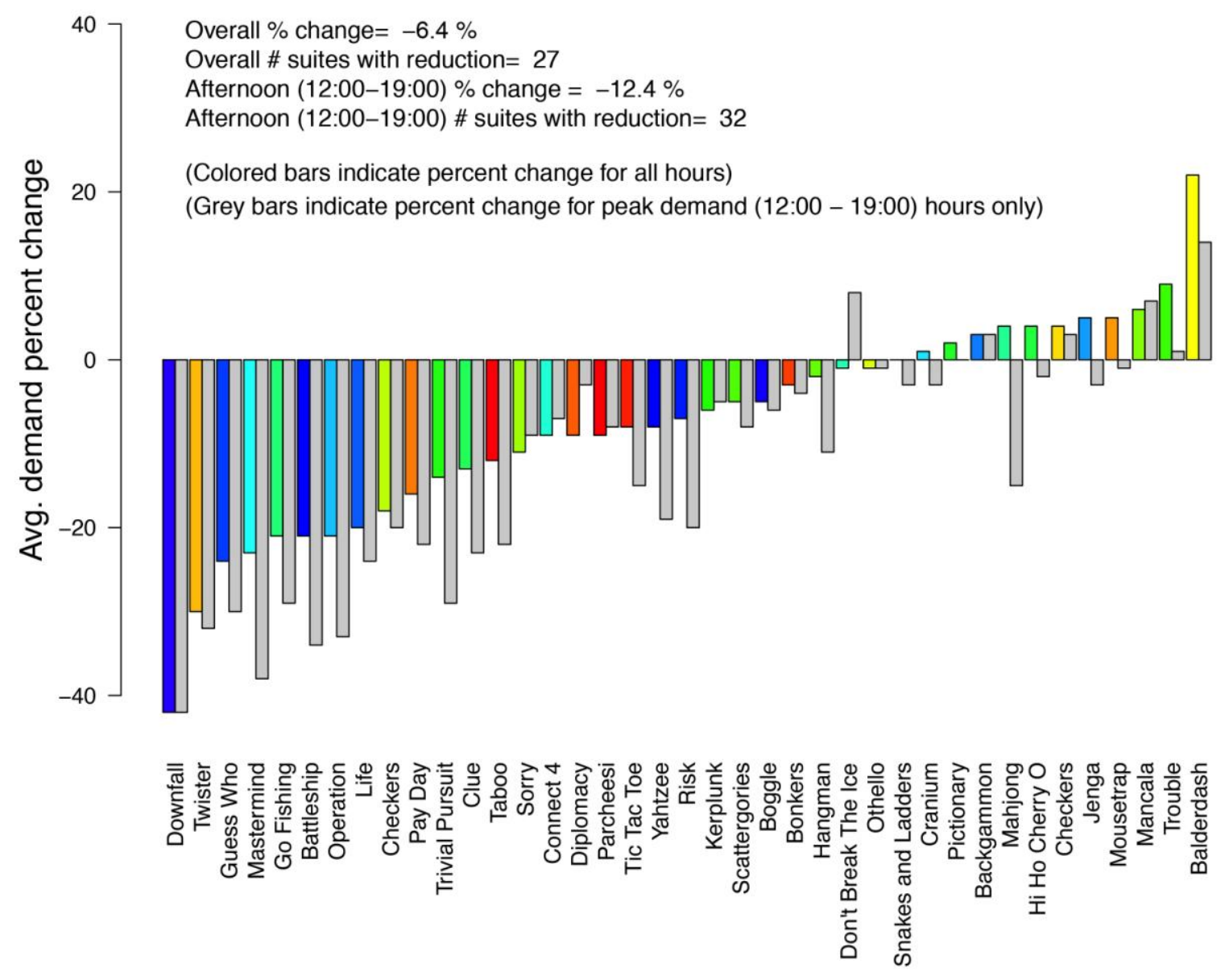

Figure 6. Competition vs. baseline.

\subsubsection{Two weeks after competition vs. baseline}

Suite-level interval data from the two weeks following the end of the competition were examined to assess whether or not individual suites "snapped back" to pre-competition (baseline) levels, a commonly reported concern for short-term behavioral interventions. As noted in section 2.3, the two-week interval was suitable for direct comparison because comparative metrics of average $\mathrm{CDD}$ and average temperature were similar to the baseline period. Results show that electricity demand for the majority of suites continued to decline, rather than "snap back" to pre-competition levels (figure 7). 


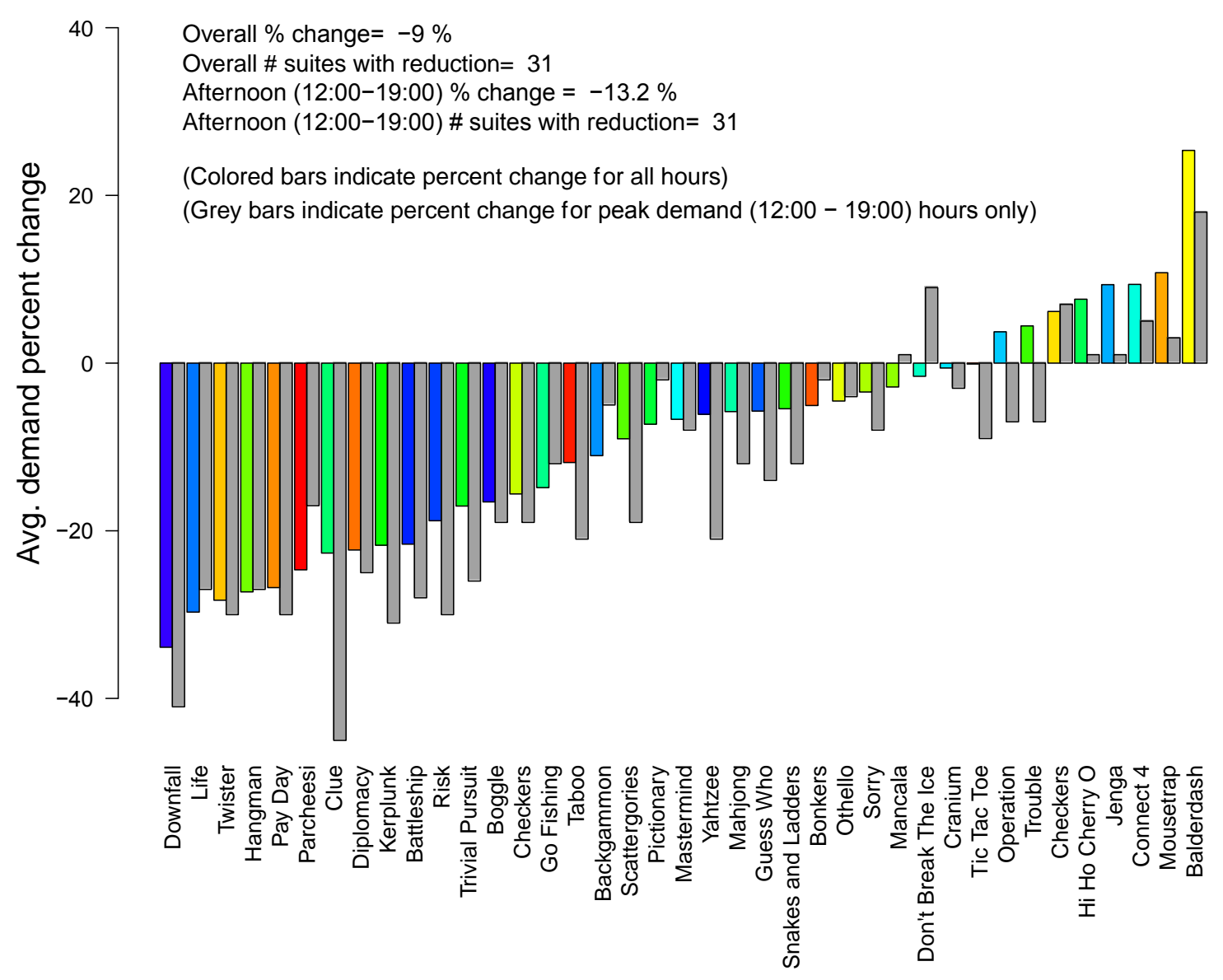

Figure 7. Two weeks after competition vs. baseline.

In the two weeks after the competition, the total demand reduction (relative to baseline) improved from the $6.4 \%$ reduction level achieved during the competition to a $9 \%$ reduction. And, 31 of the 39 suites contributed, an $80 \%$ participation level, also an improvement from the competition period. Results based on a comparison of peak demand (12:00 - 19:00) hours only (grey) show a demand reduction of 13\%, but no change in participation rate.

\subsubsection{Rest of semester vs. baseline}

The remaining 8 weeks of the semester were analyzed in two-week intervals to examine the persistence of the reductions observed during and immediately after the competition and in the "2-weeks-after" period. During the final 8 weeks of the semester, suite level demand declined by $32 \%(0.93 \mathrm{~kW})$ on average relative to the baseline period (figure 4$)$. However, this outcome was largely due to a $52.4 \%(0.96 \mathrm{~kW})$ decline in HVAC demand, where much of the reduction was expected due to less extreme weather and less demand for air conditioning. Overall, lighting and plug load demand did not change significantly. The "other" load category increased by $6 \%$, however the magnitude was small $(0.03 \mathrm{~kW})$. Consequently, we focused our analysis on discerning changes in HVAC use that were not due to weather and weather-normalized results are used to differentiate rebounders from persistent reducers. Figure 9 presents a summary of suite-level outcomes over the 
semester. Numbers along the top of the figure indicate the overall percent change in weather-normalized electricity demand for each analysis period. The figure indicates the presence of a gradual "rebound" effect after the weather-normalization was applied, which begins to manifest about four weeks after the end of the competition. However, reductions for many suites continue to persist throughout additional, or all, analysis periods.

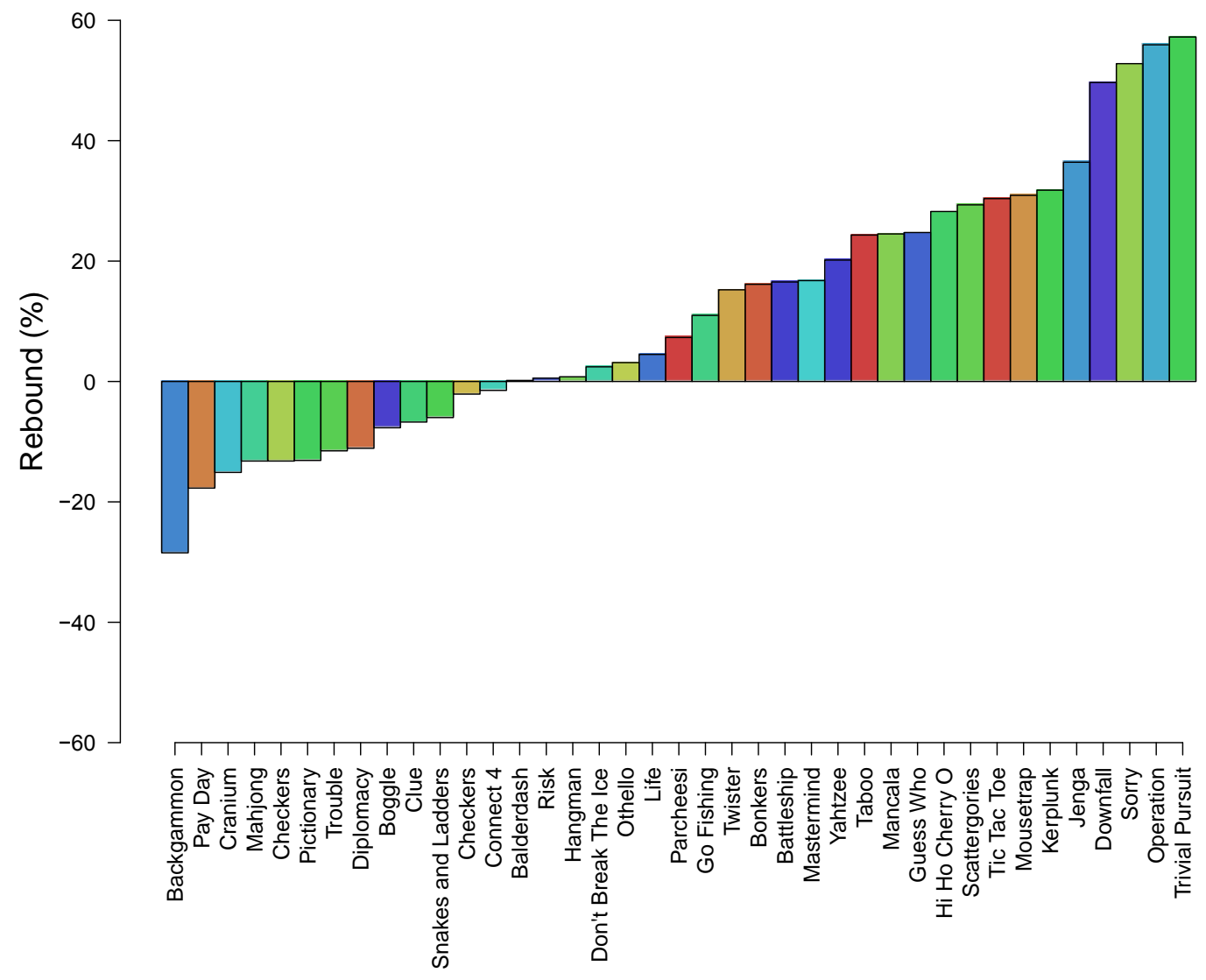

Figure 8. "Reducers" vs. "rebounders." 


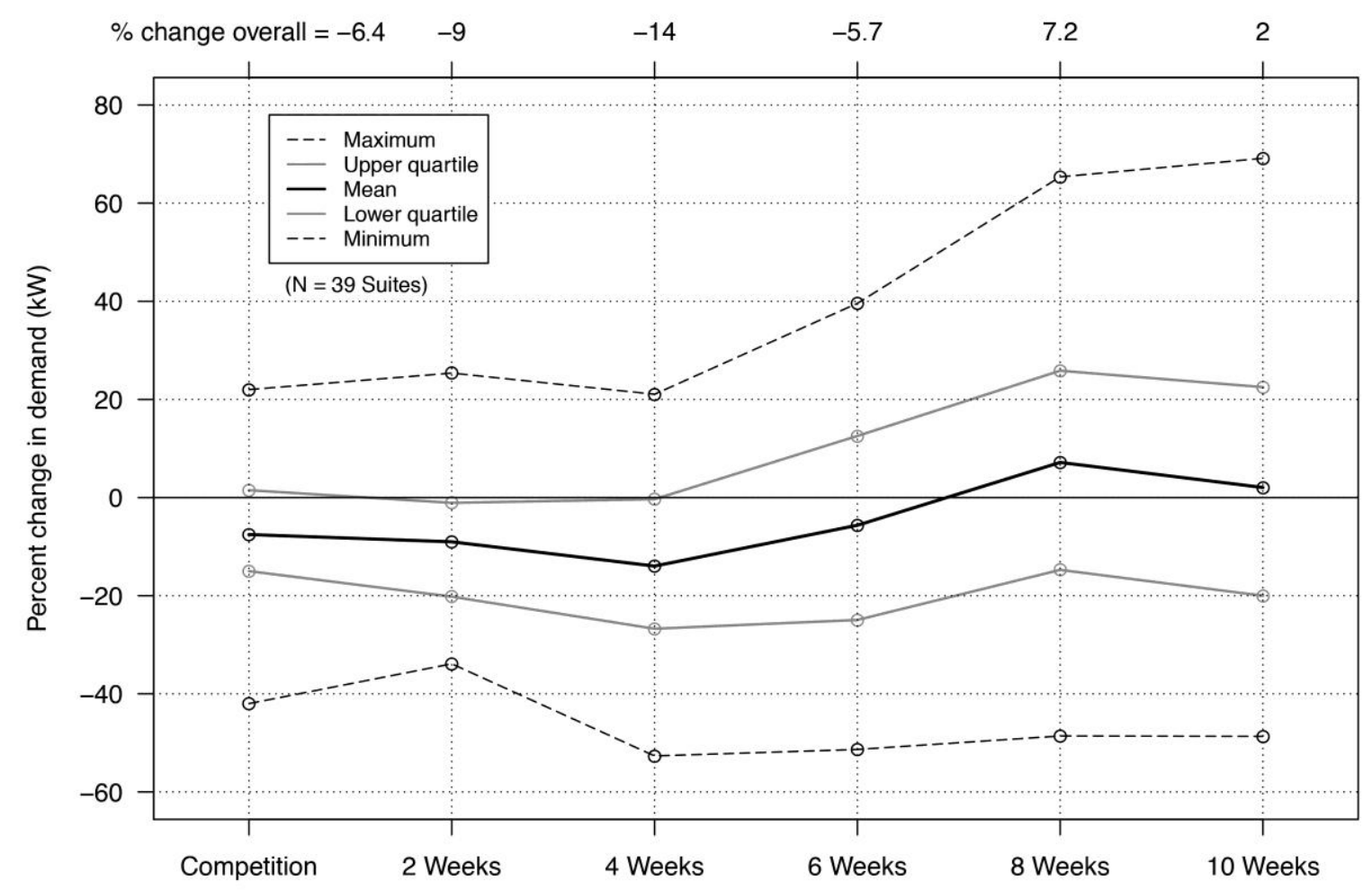

Figure 9. Weather-normalized changes in suite level electricity demand.

To quantify the magnitude of "rebound" or further reduction from competition levels, average suite-level demand achieved during the competition and for the four weeks after was compared against the average demand for the remaining six weeks of the semester. No change between the two values indicates zero rebound, and indicates that the goal of "locking in" a reduction in weather-normalized demand was achieved. A positive change indicates a rebound (or increase in demand relative to the competition), but may remain below baseline levels. A negative change indicates that the suite further reduced demand relative to the competition. A rank-order of "reducers" and "rebounders" is presented in figure 8 . Figure 8 shows that 13 suites continued to reduce, 8 suites had minor $(<10 \%)$ rebounds, and the remaining suites (19) rebounded significantly.

\subsection{Psychosocial mechanisms of changes in energy use}

To understand factors driving rebounds and further reductions from competition levels among suites, we mapped energy outcomes to suite-level self-report data. We investigated individual-level changes in several theoretically-derived variables from baseline to immediate post-intervention follow-up. We observed a significant increase in the overall frequency of self-reported PEBs performed from baseline to follow-up $(\mathrm{p}<.05)$, as well as marginal increases in self-efficacy beliefs $(\mathrm{p}<.08)$ and group identification ( $\mathrm{p}<.07)$. For details, see Sintov et al. (in press). To further explore processes of change, we examined relationships between suite-level self-report data and energy data. Specifically, using data from 12 suites with residents who completed baseline and follow-up surveys, we computed mean suite-level change scores for self- 
reported group identification, self-efficacy, and PEB. We plotted these against suite-level changes in energy use for each of the three study time periods. See table 2 for results.

Table 2. Explaining change in energy use across three study periods based on change in psychosocial and behavioral characteristics.

\begin{tabular}{|l|l|l|l|}
\hline & $\begin{array}{l}\text { During } \\
\text { intervention }\end{array}$ & $\begin{array}{l}\text { 2-weeks post } \\
\text { intervention }\end{array}$ & $\begin{array}{l}\text { Remainder of } \\
\text { semester }\end{array}$ \\
\hline $\begin{array}{l}\text { Change in self- } \\
\text { efficacy }\end{array}$ & $\mathbf{R}^{2=.629}$ & $\mathbf{R}^{2=.297}$ & $\mathbf{R}^{2=}-.11$ \\
$\mathbf{p}<.01$ & $\mathbf{p}<.01$ & $\mathbf{R}^{2=.209}$ \\
\hline $\begin{array}{l}\text { Change in group } \\
\text { identification }\end{array}$ & $\mathbf{R}^{2=-.095}$ & $\mathbf{R}^{2=.288}$ & $\mathbf{p}<.11$ \\
\hline Change in PEB & $\mathbf{p}<.65$ & $\mathbf{p}<.07$ & $\mathbf{R}^{2=.056}$ \\
& $\mathbf{R}^{2=.133}$ & $\mathbf{R}^{2=.340}$ & $\mathbf{p}<.25$ \\
\hline
\end{tabular}

\subsubsection{Change in self efficacy}

During the intervention, changes in self-efficacy were strongly positively associated with changes in energy use. In isolation, changes in self-efficacy accounted for $62.9 \%$ of the variance in change in energy use from baseline to intervention $(\mathrm{p}<.01)$. In the two weeks following the intervention, this effect was still observed, but was weaker $\left(R^{2}=0.297\right.$, $\mathrm{p}<.06)$. Examining the remainder of the semester, the effect was no longer significant. In other words, the more self-efficacy increased over the course of the intervention, the great the energy reductions achieved during the intervention, an effect that tapered off immediately following the intervention, and eventually wore off.

\subsubsection{Change in group identification}

During the intervention, suite-level changes in group identification were not associated with changes in energy use, nor was a statistically significant effect found during later periods based on the threshold level of $(\mathrm{p}<0.05)$. However, in the two weeks following the intervention, a marginally significant effect appeared whereby the magnitude of change in group identification observed during the intervention was moderately and positively associated with energy savings $\left(\mathrm{R}^{2}=.288, \mathrm{p}<.063\right)$. Examining the remainder of the semester, the effect was slightly attenuated, but remained marginally significant $\left(\mathrm{R}^{2}=.209, \mathrm{p}<.11\right)$. In other words, a latency effect was observed, contrasting with the pattern of findings observed for self-efficacy: the more group identification increased over the course of the intervention, the greater the energy reductions achieved following the intervention.

\subsubsection{Change in Pro-Environmental Behavior (PEB)}

We mapped changes in self-reported PEB onto changes in suite-level energy use for the three time periods examined. A small and marginally significant effect was observed whereby larger increases in self-reported PEB throughout the intervention were associated with greater savings during the intervention $\left(\mathrm{R}^{2}=.133, \mathrm{p}<.10\right)$. In the two weeks after the intervention, this effect became much stronger, explaining approximately one-third of the variance in change in energy demand $(\mathrm{p}<.05)$. For the remainder of the semester, however, the effect was small and nonsignificant $\left.\mathrm{R}^{2}=.056, \mathrm{p}<.26\right)$. The findings 
suggest that during the intervention, as PEB increased, energy savings increased accordingly, lending support to the use of self-report methods in this study. That PEB increases made during the intervention were more strongly associated with energy savings immediately following the intervention may suggest a partial latency effect whereby the patterns of change in PEB observed during the intervention continued and increased post-intervention, including energy saving behaviors which were reflected in the suite-level energy savings. The changes however did not persist throughout the entire semester, suggesting that engagement with the intervention stimuli may have been key in driving the results.

\subsection{Understanding the rebound effect}

We sought to understand not only changes in energy use over time, but also the psychosocial correlates underlying such changes. Significant relationships were found for both baseline self efficacy scores, and change in self-efficacy scores between baseline and follow-up. In contrast to our findings for the relationship between change in self efficacy and short term reductions (section 3.2.1), where increases in self-efficacy were strongly related to demand reduction, in this case, both greater baseline and change in self efficacy are related to greater rebound effects. These findings raise the question of students' motivation for engaging in savings behaviors during the intervention. Participation may have been motivated in large part by the competition - an external factor tied to tangible rewards. Mapping self-efficacy onto this, growth in self-efficacy may have been tied more closely to competing and winning rather than the ability to make an impact on building energy savings. In addition, it is unknown how self-efficacy evolved after the competition ended, and the impact of the competition results on residents' behavior. Self- efficacy may have declined among residents who competed but did not win, as they did not receive any reinforcement for their efforts. This may have been particularly fitting if we consider motivation for participating - among those whose primary motivation was winning, rather than to save energy for intrinsic or other reasons, losing may have adversely impacted their energy savings behaviors. We did not gather self-report data beyond the follow-up survey, so future research is needed to further investigate this question. Indeed, prior research has found that self-efficacy can decrease when individuals' efforts to save are not reflected in unit- or building-level energy use [33]. To mitigate this potential negative impact, a recent review of competition-based energy reduction interventions recommended focusing on good performance rather than winning per se, and also allowing for multiple winners when possible [33]. As an alternative explanation, it is also possible that raising self-efficacy is not sufficient for generating sustained saving, and other factors took over following the intervention.

\section{CONCLUSIONS}

In our study, a relatively short-term competition-based intervention led to significant reductions in overall and peak energy use, and these reductions persisted beyond the intervention period. However, the rebound effect that gradually emerged among a subset of participants suggests that persistent engagement may be needed to maximize the potential of occupant participation. We observed reductions during peak demand hours $(12: 00-19: 00)$ during the competition $(12 \%$ reduction) that persisted over the following two weeks of peak outdoor temperatures (13\% reduction), despite an end to the 
competition and all related incentives. Roughly one-quarter of suites achieved reductions greater than $20 \%$ during peak demand hours. Given the importance of load management, especially in southern California, this is a particularly important finding suggesting that competition-based interventions have the potential achieve even greater reductions if effective outcomes at the suite level can be better understood and scaled.

We demonstrate that psychosocial factors can help to explain both short and long-term changes in energy use, with implications for the design of future competitions and refinement of sociotechnical energy and demand management systems. Increases in both self-efficacy and group identification explained reductions, although these changed over time: While initial reductions were associated with increased self efficacy, increases in group identification were more strongly associated with energy reductions over longerterm follow-up. These findings supply information for creating appropriate "energy levers"; building operators can use this information to improve buildings and energy management program design that incorporates accessible levers. Such findings may improve the ability of knowledge-based systems to forecast participation rates and betterpredict the magnitude and duration of demand reduction achievable from short-term, competition-based interventions. This study is a first step in exploring the effectiveness of short term, behaviorally driven interventions on long-term changes to whole-building energy consumption and peak demand.

\subsubsection{Limitations and future directions}

This research should be viewed in light of several limitations. First, our intervention approached combined multiple tools of behavior change, so it was not possible to attribute observed changes to any one in particular. Future study designs should provide for disentangling the contributions of individual intervention components. Also, given the high attrition rate from baseline to follow-up, findings involving survey data should be interpreted with caution. Third, we did not collect self-report data beyond the end of the intervention period; future work should provide for such assessment to examine longterm relationships between energy use and psychosocial motivators of change.

A final limitation was that we leveraged circuit-level interval data to discern the end use types and time-of-use patterns with the greatest potential for occupants with PEB. In our test building, the observed reductions (overall and peak) were driven almost entirely by reductions in HVAC usage. Although we were able to model patterns in HVAC energy demand in response to outdoor temperatures, we did not monitor interior suite temperatures to quantify and model the indoor thermal conditions tolerated (or preferred) by residents. This information would be highly valuable in modeling occupant behavior in building energy modeling scenarios to calculate the overall and peak load reductions achievable while maintain high levels of occupant acceptance. Techniques are available to collect detailed physical and subjective thermal data in-situ at an occupant level [16], however these techniques are challenging to scale to whole building user populations. We plan to collect participant subjective feedback using mobile devices paired with indoor temperature measurements in future testing to address this limitation. 


\section{ACKNOWLEDGMENTS}

This work was funded by the US Department of Energy Grant \#DE-OE0000192 and Los Angeles Department of Water and Power (LADWP). Incentives were funded by the USC Office of Residential Life. The authors would like to thank Jack Trump, Sally

Behpoornia, and their staff of Residential Advisors for 2014-2015 for their integral roles in implementing the project; Carol Fern and Chris Bovia at USC Facilities Management Services for overseeing meter and kiosk installation and providing electricity and weather data; and Ellen Dux, Agassi Tran, Bingye Chen, Catherine Swaidan, and Arash Zadeh for assisting in materials development and data entry; and Adam Chhan and Justin Powels at LADWP for their advice. 


\section{REFERENCES}

[1] Abrahamse, W., \& Steg, L. (2013). Social influence approaches to encourage resource conservation: A meta-analysis. Global Environmental Change, 23(6), 1773-1785.

[2] Abrahamse, W., Steg, L., Vlek C., \& Rothengatter, T. (2005). A review of intervention studies aimed at household energy conservation. Journal of Environmental Psychology, 25, 273-290.

[3] Ajzen, I. (1991), "The theory of planned behavior", Organizational Behavior and Human Decision Processes, Vol. 50 No. 2, pp. 179-211.

[4] Asensio, O. I., \& Delmas, M. A. (2015), "Nonprice incentives and energy conservation", Proceedings of the National Academy of Sciences, Vol. 112 No. 6, pii. 201401880

[5] Bekker, M. J., Cumming, T. D., Osborne, N. K., Bruining, A. M., McClean, J. I., \& Leland, L. S. (2010), "Encouraging electricity savings in a university residential hall through a combination of feedback, visual prompts, and incentives", Journal of applied behavior analysis, Vol. 43 No. 2, pp. 327-331.

[6] Brewer, R. S. (2013), "Fostering sustained energy behavior change and increasing energy literacy in a student housing energy challenge", Doctoral dissertation, University of Hawai'i at Manoa.

[7] California Independent Systems Operator. (2013), "Demand response and energy efficiency roadmap: Maximizing preferred resources," available at:

http://www.caiso.com/Documents/DR-EERoadmap.pdf/ (accessed 4 April 2014).

[8] California Public Utilities Commission. (2008). California long term energy efficiency strategic plan.

[9] Campus Conservation Nationals (2014), available at: http://www.competetoreduce.org/2014/ (accessed 7 February 2015).

[10] Darby, S. (2001), "Making it obvious: designing feedback into energy consumption", in Energy efficiency in household appliances and lighting, Springer, Berlin, Heidelberg, pp. 685-696.

[11] Deci, E.L., \& Ryan, R.M. (1985), Intrinsic motivation and self-determination in human behavior, Platinum Press, Brooklyn, NY.

[12] Ehrhardt-Martinez, K, Donnelly K., \& Laitner, J. (2010). Advanced Metering Initiatives and residential feedback programs: A meta-review for household electricitysaving opportunities. Washington, D.C.: American Council for an Energy-Efficient Economy. Retrieved from http://www.aceee.org/research-report/e105 
[13] Fischer, C. (2008), "Feedback on household electricity consumption: a tool for saving energy?", Energy efficiency, Vol. 1 No. 1, pp. 79-104.

[14] Huijts, N.M.A., Molin, E.J.E., \& Steg, L. (2012). Psychological factors influencing sustainable energy technology acceptance: A review-based comprehensive framework. Renewable and Sustainable Energy Reviews, 16, 525-531.

[15] Ingle, A., Moezzi, M., Lutzenhiser, L., \& Diamond, R. (2014), “Better home energy audit modelling: Incorporating inhabitant behaviours", Building Research \& Information, Vol. 42 No. 4, pp. 409-421. doi:10.1080/09613218.2014.890776.

[16] Konis, K. (2015). Leveraging Ubiquitous Computing as a Platform for Collecting Real-time Occupant Feedback in Buildings. Intelligent Buildings International. Volume 5, Issue. 3, April 2013, Pages 150 - 161.

[17] LaCommare, K.H., and Eto, J.H. (2004), "Understanding the cost of power interruptions to U.S. electricity consumers", Berkeley, CA: Lawrence Berkeley National Laboratory.

[18] McClelland, L, \& Cook, S.W. (1980b), "Energy conservation in university buildings encouraging and evaluating reductions in occupants' electricity use", Evaluation Review, Vol. 4 No. 1, pp. 119-133.

[19] Molina, M. (2014). The best value for America's energy dollar: A national review of the cost of utility energy efficiency programs. Washington, D.C.: American Council for an Energy-Efficient Economy: Retrieved March 20, 2014, from http://aceee.org/researchreport/u1402

[20] Nolan, J. M., Schultz, P. W., Cialdini, R. B., Goldstein, N. J., \& Griskevicius, V. (2008), "Normative social influence is underdetected", Personality and social psychology bulletin, Vol. 34 No. 7, pp. 913-923.

[21] Parece, E. T., Younos, T., Grossman, L. S., \& Geller, E. S. (2013), “A study of environmentally relevant behavior in university residence halls", International Journal of Sustainability in Higher Education, Vol. 14 No. 4, pp. 466-481. doi:10.1108/IJSHE-012012-0008.

[22] Petersen, J.E., Shunturov, V., Janda, K., Platt, G., \& Weinberger, K. (2007), "Dormitory residents reduce electricity consumption when exposed to real-time visual feedback and incentives," International Journal of Sustainability in Higher Education, Vol. 8 No. 1, pp. 16-33.

[23] Schultz, W. P., Nolan, J. N., Cialdini, R. B., Goldstein, N. J., \& Griskevicius, V. (2007), "The constructive, destructive, and reconstructive power of social norms", Psychological Science, Vol. 18 No. 5, pp. 429-434. 
[24] Senbel, M., Ngo, V. D., \& Blair, E. (2014), "Social mobilization of climate change: University students conserving energy through multiple pathways for peer engagement", Journal of Environmental Psychology, Vol. 38, pp. 84-93.

doi:10.1016/j.jenvp.2014.01.001.

[25] Sintov, N.D. \& Schultz, P.W. (2015). Unlocking the potential of smart grid technologies with behavioral science. Frontiers in Psychology, 6. doi:

10.3389/fpsyg.2015.00410

[26] Sintov, N.D., Dux, E., Tran, A., \& Orosz, M. (in press). What goes on behind closed doors? How college dormitory residents change to save energy during a competitionbased energy reduction intervention. International Journal for Sustainability in Higher Education.

[27] Skinner, B.F. (1953). Science and human behavior. New York: Macmillan.

[28] Steg, L., \& Vlek, C. (2009). Encouraging pro-environmental behaviour: An integrative review and research agenda. Journal of Environmental Psychology, 29, 309317

[29] Tiedmann, K., Sulyma, I., \& Rebman, M. (2007). Measuring the impact of time of use rates on peak and off-peak energy consumption: Some results from a randomized controlled experiment. Proceedings of the 2007 International Energy Program Evaluation Conference, pp. 77-87. Chicago IL: International Energy Program Evaluation Conference.

[30] United States Energy Information Administration (2015), "Electricity survey-level detail data files", available at http://www.eia.gov/electricity/data/detail-data.html/ (accessed 31 January 2015).

[31] United States Energy Information Administration (2014a), "US EIA annual energy outlook 2014", available at: http://www.eia.gov/forecasts/aeo/pdf/tbla19.pdf/ (accessed 12 February 2015)

[32] United States Federal Energy Regulatory Commission. (2009). A national assessment of demand response potential. Retrieved March 18, 2014, from http://www.ferc.gov/legal/staff-reports/06-09-demand-response.pdf

[33] Vine, E., \& Jones, C. (2015). A review of energy reduction competitions: What have we learned? California Institute for Energy and Environment (CIEE) report. Retrieved from http://uc-ciee.org/behavior-decision-making/1/721/99/nested

[34] Wood, W., Witt, G., \& Tam, L. (2005), "Changing circumstances, disrupting habits", Journal of Personality and Social Psychology, Vol. 88 No. 6, pp. 918-933. 